\title{
Le système complexe des récepteurs GABA/benzodiazépine
}

L'acide $\gamma$ aminobutyrique (GABA) est le principal neurotransmetteur inhibiteur du système nerveux central. Il exerce ses effets biologiques par l'intermédiaire de plusieurs types de récepteurs, dont les mieux caractérisés sont appelés récepteur $\mathbf{G A B A}_{\mathrm{A}}$ et $\mathbf{G A B A}_{\mathrm{B}}$. Le premier est directement associé à un canal de chlore, alors que le second est indirectement couplé à un canal cationique. Le récepteur $\mathbf{G A B A}_{A}$, dont il existe plusieurs sous-types, est également associé à un récepteur pour les benzodiazépines. Le clonage des ADNc codant pour les différentes sous-unités du récepteur GABA $_{A}$ a permis d'identifier quatre types de sous-unités $\alpha, \beta, \gamma^{2}$ et $\delta$. La forme active semble constituée de cinq sous-unités : deux sous-unités $\alpha$, deux sous-unités $\beta$ et soit une sousunité $\gamma^{2}$, soit une sous-unité $\delta$. Seul le pentamère incluant $\gamma^{2}$ semble capable de fixer les benzodiazépines. La multiplicité des isoformes de récepteur GABA permet d'expliquer la diversité des effets biologiques de ce neurotransmetteur en différentes zones du cerveau.

\section{Elisabeth Bacon Françoise Viennot}

ADRESSE

E. Bacon : chargée de recherche à l'Inserm. F. Viennot : chercheur en formation. Centre de neurochimie du Cnrs et Inserm U. 44, 5, rue Blaise-Pascal, 67084 Strasbourg Cedex, e GABA (acide gammaaminobutyrique) est le principal neuromédiateur inhibiteur du système nerveux central. Il est présent dans toutes les zones du cerveau et est actif sur environ 30 à $40 \%$ des synapses du système nerveux central [1]. Le GABA joue un grand rôle dans la neurotransmission et son récepteur a fait l'objet de nombreux travaux. Trop souvent encore, de nombreux auteurs emploient indifféremment les termes de "récepteur GABA/benzodiazépine " ou "récepteur GABA " pour désigner ce qui est actuellement connu sous le terme de récepteur $\mathrm{GABA}_{\mathrm{A}}$. Or il existe une deuxième sous-classe de récepteurs dénommés sites $\mathrm{GABA}_{\mathrm{B}}$ qui n'a été réellement bien décrite qu'en
1980 [2]. Plus récemment encore, une troisième classe de récepteurs GABA a été mise en évidence [3]. Ces sous-classes de récepteurs lient toutes le GABA et conduisent toutes à l'inhibition neuronale. Le problème qui se pose est donc d'identifier les éléments qui différencient un récepteur $\mathrm{GABA}_{\mathrm{A}}$ d'un récepteur $\mathrm{GABA}_{\mathrm{B}}$, étant donné qu'ils induisent le même effet avec le même ligand.

La structure du récepteur $\mathrm{GABA}_{\mathrm{A}}$ est bien connue : il comprend un site de liaison spécifique pour le GABA et des sites pour diverses substances (en particulier les benzodiazépines, molécules de grand intérêt thérapeutique), sites qui fonctionnent de manière allostérique [4-5]. Il est directement associé à un canal ioni- 
que (canal chlore). C'est donc un récepteur ionotrope qui appartient à la classe 1 de la super-famille des récepteurs.

Les informations concernant le récepteur $\mathrm{GABA}_{\mathrm{B}}$ sont plus succinctes : il ne comporte que le site de liaison du GABA. C'est un récepteur métabotrope (associé à distance à un canal cationique par l'intermédiaire d'un système de messagers secondaires) et il peut donc être rattaché à la classe 2 de la super-famille des récepteurs (figure 1) [6]. Cette revue considérera plus en détail le récepteur $\mathrm{GABA}_{\mathrm{A}}$, qui est le récepteur GABA " classique " et le mieux connu actuellement, après avoir défini les caractéristiques distinctives des autres récepteurs GABA.

\section{Propriétés distinctives des différents sites GABA et effecteurs spécifiques}

La subdivision du récepteur GABA en deux sous-types est consécutive à la découverte d'une population de sites GABA présentant une insensibilité à la bicuculline (antagoniste du GABA). Sur ces sites, le $\left[{ }^{3} \mathrm{H}\right] \mathrm{GABA}$ lié n'est pas déplacé par la bicuculline ; deux sous-classes de récepteurs se distinguent donc: les sites bicuculline-sensibles " classiques " ou sites $\mathrm{GABA}_{\mathrm{A}}$ et les sites bicucullineinsensibles ou sites $\mathrm{GABA}_{B}$ qui tous deux lient le GABA et induisent une neurotransmission inhibitrice [2]. Par ailleurs, le ( - )baclofène, analogue du GABA, est inactif sur les sites $\mathrm{GABA}_{\mathrm{A}}$ mais se lie aux sites $\mathrm{GABA}_{B}$. Ainsi, la liaison spécifique de $\left[{ }^{3} \mathrm{H}\right] \mathrm{GABA}$ pourra être étudiée sur les sites $\mathrm{GABA}_{\mathrm{A}}$ en présence de baclofène (pour bloquer la liaison spécifique sur les sites $\mathrm{GABA}_{\mathrm{B}}$ ); les sites $\mathrm{GABA}_{\mathrm{B}}$, quant à eux, seront révélés en présence de $\mathrm{CaCl}_{2}$ et de bicuculline (cette dernière bloquant la liaison spécifique sur les sites $\mathrm{GABA}_{\mathrm{A}}$ [7]. Les benzodiazépines et les barbiturates n'interagissent pas avec le récepteur $\mathrm{GABA}_{\mathrm{B}}$. D'autres éléments distinctifs, spécifiques des récepteurs $\mathrm{GABA}_{\mathrm{A}}$ et $\mathrm{GABA}_{\mathrm{B}}$, ont été identifiés et utilisés depuis et sont présentés dans le Tableau I. Le récepteur $\mathrm{GABA}_{\mathrm{B}}$ peut mettre en jeu, selon sa localisation, deux types d'effecteurs distincts avec activation $\mathrm{m} / \mathrm{s} n^{\circ} 8$, vol. 6 , octobre 90

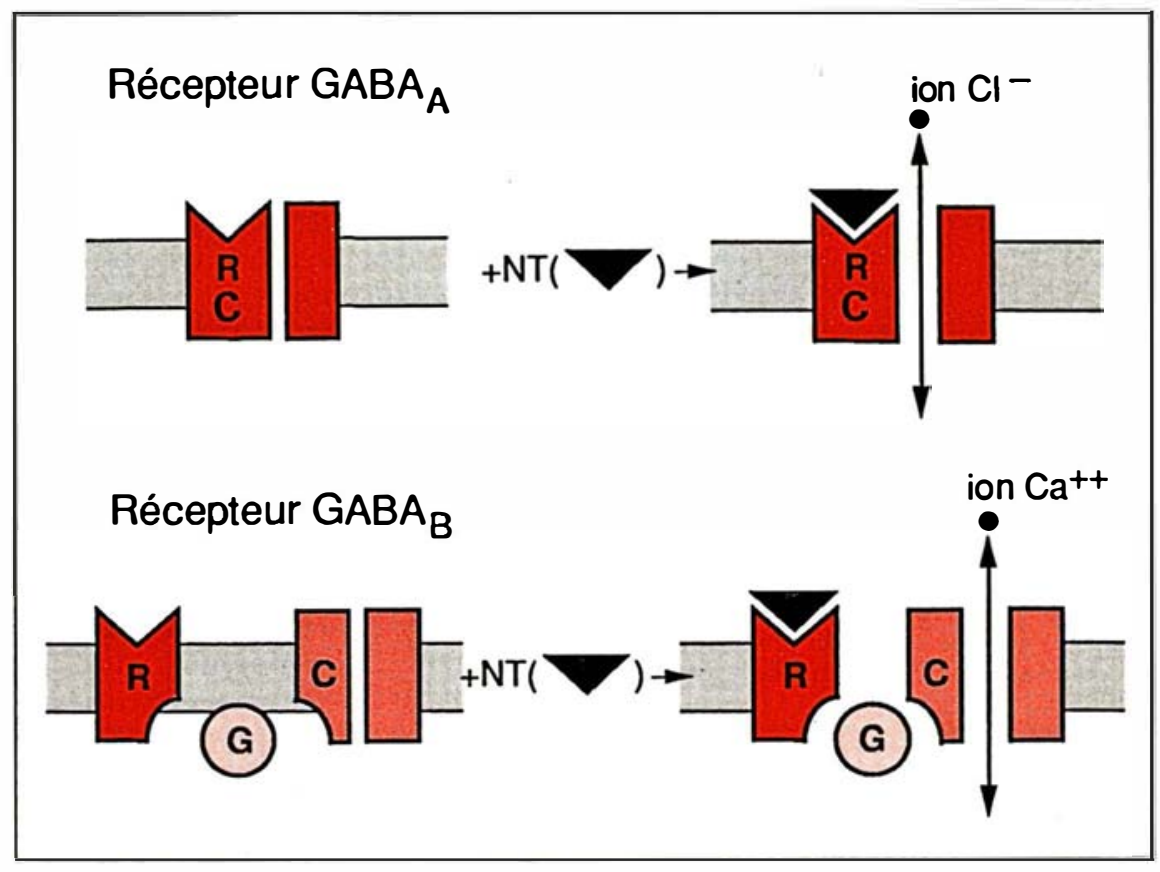

Figure 1. Les récepteurs $\mathbf{G A B A}_{\mathbf{A}}$ et $\mathbf{G A B A}_{B}$. Récepteur $G A B A_{A}$ : le récepteur $(R)$ et le canal ionique (C) font partie de la même molécule. Le neurotransmetteur (NT), une fois fixé sur cette molécule, ouvre le canal ionique. Récepteur $G A B A_{B}$ : le récepteur est couplé au canal ionique par l'intermédiaire $d^{\prime}$ une protéine $G$. (D'après [6].)

Tableau I

PROPRIÉTÉS DES SYSTĖMES RÉCEPTEURS GABA $A_{A}$ ET GABA

\begin{tabular}{|c|c|c|}
\hline & $\mathrm{GABA}_{A}$ & $\mathrm{GABA}_{B}$ \\
\hline Agonistes & $\begin{array}{l}\text { GABA } \\
\text { muscimol } \\
\text { 3-APS }\end{array}$ & $\begin{array}{l}\text { GABA } \\
\text { 1-) baclofène } \\
\text { 3-APA }\end{array}$ \\
\hline Antagonistes & bicuculline & phaclofène \\
\hline \multicolumn{3}{|c|}{ Modulation de la liaison spécifique } \\
\hline $\begin{array}{l}\text { Dépendance vis-à-vis des ions } \\
\text { Effet du GTP }\end{array}$ & & $\begin{array}{l}\mathrm{Ca}^{++} \text {et/ou } \mathrm{Mg}^{++} \\
\text {Diminution }\end{array}$ \\
\hline \multicolumn{3}{|c|}{ Type de conductance modulée } \\
\hline & $\mathrm{Cl}^{-}$(positivement) & $\begin{array}{l}\mathrm{Ca}^{++} \\
\text {(négativement) } \\
\mathrm{K}^{+} \text {(positivement) }\end{array}$ \\
\hline
\end{tabular}

3-APS : acide 3-aminopropyl sulfonique; 3-APA : acide 3-aminopropyl phosphorique. 


\section{RÉFÉRENCES}

1. Matsumoto RR. GABA receptors : are cellular differences reflected in function? Brain Res Rev 1989 ; 14: 203-25.

2. Bowery NG, Hill DR, Hudson AL, et al. ( - Baclof en decreases neurotransmitter release in the mammalian CNS by an action at a novel GABA receptor. Nature $1980 ; 283$ : $92-4$

3. Drew CA, Johnston GAR, Weatherby RP. Bicuculline-insensitive GABA receptors: studies on the binding of (-)baclof en to rat cerebellar membranes. Neuroscience 1984 ; 52 : 317-21.

4. Dingemanse J, Breimer DD. Benzodiazepine receptors. Pharmacy Int 1984; 5 : 33-6.

5. Olsen R, Yang J, King RG, Dilber A, Stauber GB, Ransom RW. Barbiturate and benzodiazepine modulation of GABA receptor binding and function. Life Sci 1986; 39 : 1969-76.

6. Bockaert J. Les protéines G étendent leur pouvoir sur les canaux ioniques. médecine/sciences $1989 ; 5: 562-9$.

7. Yamada I, Hirata A, Nakahiro M, Yoshida $H$. Endogenous inhibitor of $\mathrm{GABA}_{\mathrm{B}}$ and $\mathrm{GABA}_{\mathrm{A}}$ receptors. Neurochem Int 1987 $10: 65-70$.

8. Feltz A, Demeneix B, Feltz $\mathrm{P}$, et al. Intracellular effectors and modulators of $\mathrm{GABA}_{\mathrm{A}}$ and $\mathrm{GABA}_{\mathrm{B}}$ receptors : a commentary. Biochimie 1987 ; 69 : 395-406. 9. Stephenson FA. Understanding the $\mathrm{GABA}_{A}$ receptor : a chemically gated ion channel. Biochem J 1988 ; 249 : 21-32.

10. Bowery NG, Price GW, Hudson AL, Hill DR, Wilkin GP, Turnbull MJ. GABA receptor multiplicity. Visualization of different receptor types in the mammalian CNS. Neuropharmacol 1984 ; 23 : 219-31.

11. Sieghart W. Multiplicity of $\mathrm{GABA}_{\mathrm{A}}$ benzodiazepine receptors. Trends Pharmacol Sci 1989 ; 10 : 407-11.

12. Ito $Y, \operatorname{Lim} \mathrm{DK}$, Hoskins $B$, Ho IK. Bicuculline up-regulation of $\mathrm{GABA}_{\mathrm{A}}$ receptors in rat brain. $J$ Neurochem $1988 ; 51$ : 145-52.

13. Kuriyama K, Taguchi JI. Purification of gamma-aminobutyric acid receptor, benzodiazepine receptor and $\mathrm{Cl}$ channel from bovine cerebral cortex by benzodiazepine affinity gel column chromatography. Neurochem Int $1987 ; 10$ : 253-63.

14. Fry JP, Rickets C, Biscoe TJ. On the location of gamma-aminobutyrate and benzodiazepine receptors in the cerebellum of the normal $\mathrm{C} 3 \mathrm{H}$ and lurcher mutant mouse. Neurosci 1985 ; 14 : 1091-101.

15. Guidotti A, Gale K, Suria A, Toffano G. Biochemical evidence for two classes of GABA receptors in rat brain. Brain Res $1979 ; 172: 566-71$

16. Maksay G. GABA $A_{A}$ receptor populations bind agonists and antagonists differentially and with opposite affinities. $J$ Neurochem 1988 ; 50 : 1865-71.

17. Moffet JR, Namboodiri A, Neale JH. Two forms of the $\mathrm{GABA}_{\mathrm{A}}$ receptor distinguished by anion-exchange chromatography. FEBS Lett 1989 ; 247 : 81-5.

18. Kardos J, Maderspach K, Simonyi M. Towards a more physiological approach in GABA binding. Neurochem Int 1985; 7 : 737-43. d'une perméabilité potassique et/ou blocage des entrées calciques [8]. Par ailleurs, il est généralement admis que les récepteurs $\mathrm{GABA}_{\mathrm{A}}$ sont majoritairement post-synaptiques, alors que l'on trouve les récepteurs $\mathrm{GABA}_{\mathrm{B}}$ aux niveaux à la fois préet post-synaptiques [1, 9]. D'une manière générale, les récepteurs $\mathrm{GABA}_{\mathrm{B}}$ sont moins abondants que les récepteurs $\mathrm{GABA}_{\mathrm{A}}$ dans le système nerveux central et, de plus, la distribution des deux types de récepteurs est différente. Bien qu'ils puissent être présents simultanément dans de nombreuses régions du cerveau, il est des zones où l'on ne trouve qu'un seul type : ainsi, les récepteurs $\mathrm{GABA}_{\mathrm{A}}$ sont seuls présents dans la lamina molecularis du bulbe olfactif et la couche granulaire du cervelet, cependant que les sites $\mathrm{GABA}_{\mathrm{B}}$ sont présents dans le nucleus interpedoncularis sans que l'existence de sites $\mathrm{GABA}_{\mathrm{A}}$ y ait été mise en évidence [10].

Enfin, il existerait une troisième classe de récepteurs $\mathrm{GABA}$, le récepteur $\mathrm{GABA}_{\mathrm{C}}$ qui est stimulé par le GABA mais insensible à la fois à la bicuculline et au baclofène [3].

\section{- Le récepteur GABA}

Il s'agit du récepteur GABA " classique ". C'est un complexe macromoléculaire comportant, dans sa forme la plus complète, des sites de reconnaissance pour le GABA, les benzodiazépines, la picrotoxine (et autres agents convulsivants) et les barbiturates, ces deux derniers étant localisés sur un canal chlore figure 2 [4]). Chacun de ces types de sites de fixation peut interagir allostériquement avec un ou plusieurs des autres types : par exemple, le récepteur $\mathrm{GABA}_{\mathrm{A}}$ est stimulé par divers agonistes (GABA, muscimol) et inhibé de façon compétitive par la bicuculline (antagoniste du GABA) et non compétitive par la picrotoxine qui bloque le canal chlore [1, 10].

Le site $\mathrm{GABA}_{\mathrm{A}}$ lui-même se subdivise en plusieurs sous-classes. On reconnaît généralement deux populations de récepteurs $\mathrm{GABA}_{\mathrm{A}}$ : le site à haute affinité est unanimement reconnu tandis qu'un site à basse affinité n'est pas mentionné par tous les auteurs [1,11] ; tous s'accordent cependant sur une valeur de l'ordre du nanomolaire pour la constante de dissociation $\left(\mathrm{K}_{\mathrm{D}}\right)$ du complexe ligand-récepteur du site à haute affinité. Pour le site de basse affinité, les valeurs avancées sont soit de l'ordre de $10 \mathrm{nM}$ [12-13], soit de l'ordre de 100 nM [14-15]. Un site de superbasse affinité est évoqué par Maksay [16] qui définit ainsi trois souspopulations de sites $\mathrm{GABA}_{\mathrm{A}}$. Indépendamment de ces distinctions fondées sur des études de liaison spécifique, deux formes chimiques de récepteurs $\mathrm{GABA}_{\mathrm{A}}$ peuvent être distinguées après purification par chromatographie sur colonne d'affinité du récepteur solubilisé. Ces formes possèdent cependant des caractéristiques de liaison spécifique identiques pour le muscimol et le flunitrazepam [17]. Certains auteurs ont suggéré que les récepteurs $\mathrm{GABA}_{\mathrm{A}}$ liant le $\mathrm{GABA}$ lui-même et ceux liant le muscimol pourraient être des récepteurs distincts ; toutefois, les propriétés différentes observées pourraient résulter d'une portion non commune spécifique à chacun de ces ligands dans un ensemble récepteur commun [18, 19]. Enfin, le récepteur $\mathrm{GABA}_{\mathrm{A}}$ est sujet à des variations régionales puisque celui du cervelet présente des propriétés différentes de celles trouvées dans les autres zones du cerveau [20].

\section{Les récepteurs des benzodiazépines}

De nombreuses données comportementales, pharmacologiques et biochimiques, suggèrent qu'une grande partie des actions pharmacologiques des benzodiazépines se fait par l'intermédiaire de systèmes neuronaux dans lesquels le GABA est le neuromédiateur [20-21]. Les sites benzodiazépine peuvent être classés en deux catégories, à savoir les sites " centraux " et " périphériques ", qui se distinguent par leur sensibilité à l'égard de ligands spécifiques. Cette dénomination a une origine purement historique et ne présume en fait en rien de la localisation de ces sites qui peuvent tous deux se rencontrer au niveau central et/ou périphérique. Toutefois, les sites dits "centraux" sont localisés sur les neurones et les sites "périphériques" sont essentiellement présents dans la glie [22]. Ils 
ne sont pas marqués par le flunitrazepam, agoniste spécifique des récepteurs centraux, mais lient spécifiquement le ligand RO 5-4864 [23].

L'hétérogénéité des récepteurs benzodiazépine centraux a été mise en évidence par l'observation des interactions complexes de certaines classes de composés, les triazolopyridazines et les bêta-carbolines, avec les récepteurs benzodiazépine : les expériences de liaison spécifique de ces composés et de compétition avec le flunitrazepam permettent de définir deux populations de récepteurs benzodiazépine [21]. On peut ainsi distinguer les sites benzodiazépine centraux à haute et à basse affinité, nommés respectivement récepteurs de type I et II, alors que le flunitrazepam lui-même possède une affinité identique à l'égard des deux sites [21]. Ces deux classes de récepteurs se distinguent également par leurs propriétés biochimiques : le récepteur de type I est thermolabile, le site de type II étant plus stable à la chaleur. De plus, le GABA protège les sites de type II de l'inactivation thermique mais n'assure qu'une protection partielle des récepteurs de type I [21]. Le récepteur de type I n'est pas solubilisé par le Triton alors que le récepteur de type II est Tritonsoluble. Un niveau supplémentaire de complexité est introduit par certains auteurs [21] qui parlent d'un troisième site benzodiazépine d'affinité

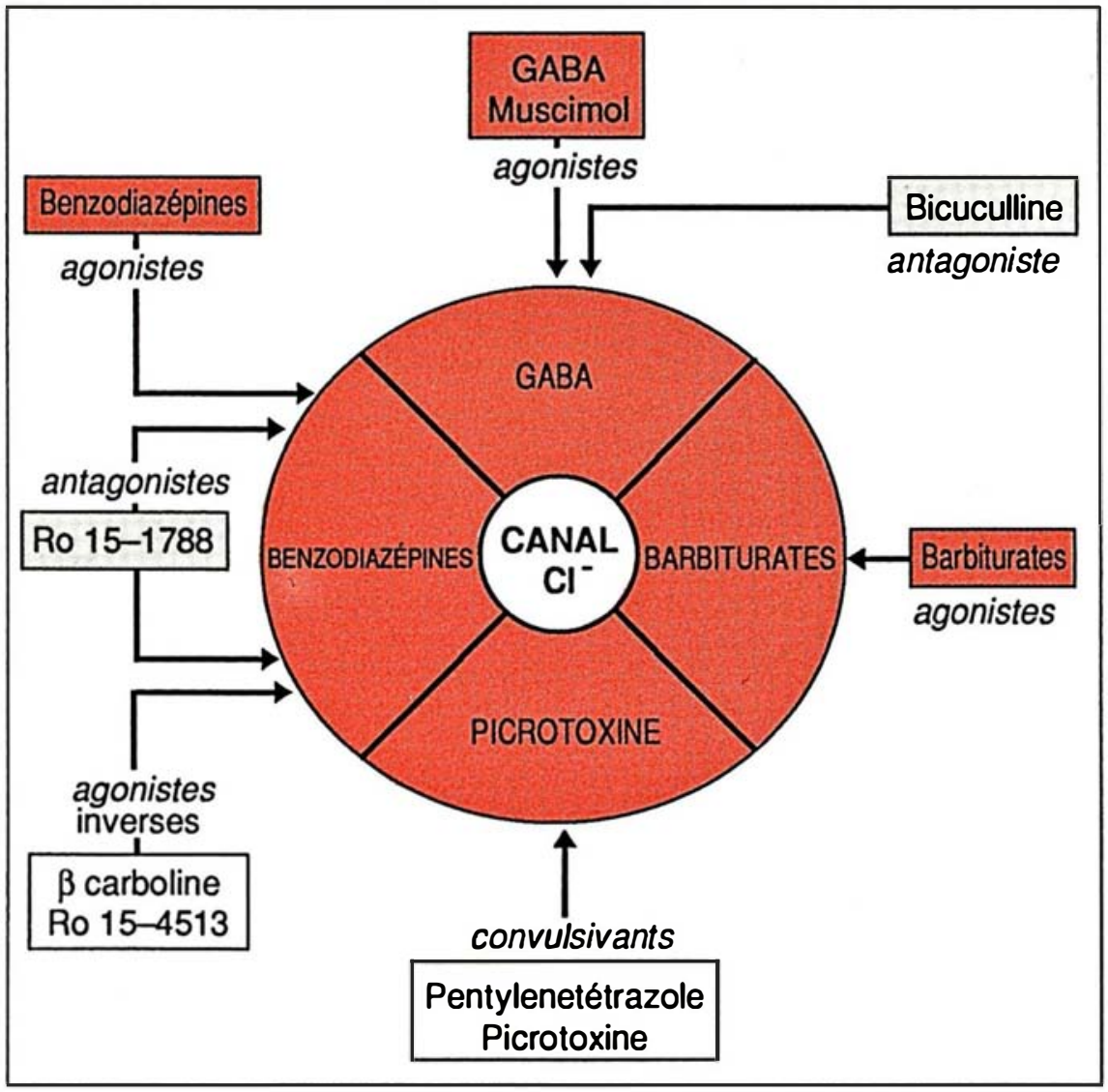

Figure 2. Le complexe récepteur GABA/benzodiazépine. Cette représentation schématique illustre les différents sites de liaison constitutifs du complexe récepteur. L'occupation du récepteur $G A B A_{A}$ par un agoniste induit l'ouverture du canal chlore ainsi qu'un changement allostérique de la conformation du site de reconnaissance des benzodiazépines, entraînant une augmentation de l'affinité du récepteur pour les benzodiazépines. Par ailleurs, les agonistes benzodiazépine augmentent l'affinité du GABA pour son site récepteur ou améliorent le couplage du récepteur $G_{A B A_{A}}$ avec le canal chlore. $R O$ 15-1788 = Flumazenil. (D'après [48].)

$\mathrm{m} / \mathrm{s} n^{\circ} 8$, vol. 6, octobre 90 dite "super-haute" $\left(\mathrm{K}_{\mathrm{D}}\right.$ de 30 à 50 picomolaire).

Les densités relatives de ces récepteurs varient selon les régions du cerveau. Ainsi, il y aurait $90 \%$ de récepteurs de type I dans le cervelet $[21,24]$ à partir duquel un seul type de complexe récepteur GABA/benzodiazépine a pu être purifié [25]. La couche superficielle du colliculus supérieur et le caudate-putamen présentent une proportion élevée de récepteurs de type II, cependant que dans l'hippocampe et d'autres zones du cortex cérébral, les deux types coexistent [21, 22, 24].

\section{Couplage des récepteurs benzodiazépine au récepteur $G_{A B A_{A}}$ : données pharmacologiques}

IL existe une modulation allostérique mutuelle entre le GABA et les benzodiazépines au niveau du complexe récepteur : ainsi, l'affinité des ligands benzodiazépine pour leur site récepteur est augmentée en présence de GABA, tandis que les agonistes des benzodiazépines augmentent l'affinité du GABA pour son site récepteur [4]. Il existerait une différence qualitative régionale des récepteurs de type I concernant leur association avec le récepteur $\mathrm{GABA}$ : en effet, les études réalisées sur le cortex cérébral et l'hippocampe suggèrent que, dans ces régions, ce sont les récepteurs benzodiazépine de type II qui sont couplés au GABA, les récepteurs de type I n'étant pas sujets à la régulation GABAergique, alors que dans le cervelet, bien qu'il soit une zone enrichie en récepteurs de type I, on observe une stimulation de la liaison spécifique des benzodiazépines par le GABA [21, 26]. La figure 3 (p. 775) rassemble les caractéristiques de ces différents récepteurs ainsi que leurs couplages.

\section{Structure du complexe récepteur GABA/benzodiazépine : une approche moléculaire}

L'isolement du complexe récepteur $\mathrm{GABA}_{\mathrm{A}}$ /benzodiazépine par chromatographie d'affinité à partir du cerveau a ouvert la porte à des 
RÉFÉRENCES

19. Madtes P Jr, Fuller G, Gregg K. Sensitivity of GABA-displaceable $\left[{ }^{3} \mathrm{H}\right]$ muscimol binding depends upon the composition of the buffering medium. Neurochem Int $1989 ; 15: 179-83$.

20. Bristow DR, Martin IL. Light microscopic autoradiographic localization in rat brain of the binding sites for the $\mathrm{GABA}_{\mathrm{A}}$ receptor antagonist $\left.{ }^{3} \mathrm{H}\right] \mathrm{SR}$ 95531: comparison with the $\left[{ }^{3} \mathrm{H}\right] \mathrm{GABA}_{\mathrm{A}}$ receptor distribution. Eur J Pharmacol 1988 ; 148 : 283-8. 21. Gee KW, Ehlert FJ, Roeske WR, Yamamura HI. Heterogeneity of benzodiazepine receptors. Handbook Neurochem 1984 ; 6 : 575-93.

22. Richards JG, Möhler H. Benzodiazepine receptors. Neuropharmacol 1984; 23 : 233-42.

23. Gehlert DR, Yamamura HI, Wamsley JK. Autoradiographic localization of " peripheral " benzodiazepine binding sites in the rat brain and kidney using $\left[{ }^{3} \mathrm{H}\right] \mathrm{RO}$ 5-4864. Eur J Pharmacol 1983 ; 95 : 329-30. 24. Giorgi O, Corda MG, Gritti I, Mariotti M, Ongini E, Biggio G. Binding sites for $\left[{ }^{3} \mathrm{H}\right] 2$-oxo-quazepam in the brain of the cat : evidence for heterogeneity of benzodiazepine recognition sites. Neuropharmacol 1989; 28 : 715-8.

25. Duggan MJ, Stephenson FA. Benzodiazepine binding site heterogeneity in the purified $\mathrm{GABA}_{\mathrm{A}}$ receptor. Eur J Pharmacol $1988: 154: 293-8$

26. Bacon E, Matsokis N, Roujansky P, de Barry J, Gombos G. Alteration of benzodiazepine receptors in mouse cerebellum following methylazoxymethanol treatment during development. Develop Brain Res $1989 ; 47: 293-7$

27. Barnard EA, Darlison MG, Seeburg P. Molecular biology of the $\mathrm{GABA}_{\mathrm{A}}$ receptor : the receptor/channel superfamily. Trends Neurosci 1987 ; 10 : 502-9.

28. Wisden W, Morris BJ, Darlison MG, Hunt SP, Barnard EA. Localization of $\mathrm{GABA}_{\mathrm{A}}$ receptor alpha-subunit mRNAs in relation to receptor subtypes. Mol Brain Res $1989 ; 5$ : 305-10

29. Séquier JM, Richards JG, Malherbe $P$, Price GW, Mathews S, Möhler H. Mapping of brain areas containing RNA homologous to cDNAs encoding the alpha and beta subunits of the rat $\mathrm{GABA}_{\mathrm{A}}$ gammaaminobutyrate receptor. Proc Nat Acad Sci USA $1988 ; 85: 7815-9$.

30. Sieghart W, Eichinger A, Zezula J. Comparison of tryptic peptides of benzodiazepine binding proteins photolabeled with $\left[{ }^{3} \mathrm{H}\right]$ Flunitrazepam or $\left[{ }^{3} \mathrm{H}\right] \mathrm{RO}$ 15-4513. $J$ Neurochem 1987; 48 : 1109-14.

31. Schoch $P$, Richards JG, Häring $P$, et al. Co-localization of $\mathrm{GABA}_{\mathrm{A}}$ receptors and benzodiazepine receptors in the brain shown by monoclonal antibodies. Nature 1985 ; 314: 168-71

32. Pritchett DB, Sontheimer $H$, Shivers $\mathrm{BD}$, et al. Importance of a novel $\mathrm{GABA}_{\mathrm{A}}$ receptor subunit for benzodiazepine pharmacology. Nature 1989 ; 338 : 582-5.

33. Shivers BD, Killisch I, Sprengel R, et al. Two novel $\mathrm{GABA}_{\mathrm{A}}$ receptor subunits exist in distinct neuronal subpopulations. Neuron 1989 ; 3 : 327-37. études structurales et génétiques de ce récepteur. Après clivage en petits peptides et détermination des structures primaires, Barnard et al. [27] ont préparé diférentes sondes nucléotidiques pour caractériser et identifier au plan moléculaire les différentes sous-unités constitutives du complexe récepteur. Ces séquences désoxyribonucléotidiques ont permis de détecter, d'enrichir et d'isoler les ADNc les plus longs correspondant aux gènes codant pour les diverses sousunités constitutives du complexe récepteur. Les ARNm correspondants soit ont été " pêchés " dans une préparation d'ARNm totaux, soit ont été synthétisés in vitro pour être ensuite injectés dans des oocytes de xénope, afin d'étudier leur expression in vitro, soit ont été reconvertis en ADNc " complets " $[28,29]$. Le marquage photochimique du récepteur (photoaffinité) permet d'étudier la molécule de récepteur isolée. Une électrophorèse en gel dénaturant permet la séparation des différentes protéines présentes, ici les sous-unités du complexe, en fonction de leur poids moléculaire apparent. Par ailleurs, les ARNm dont les produits de transcription correspondent à ces protéines sont analysées en Northern blot et la taille des ARNm ainsi obtenus permet une bonne estimation du poids moléculaire de la protéine récepteur après transcription [30].

Les expériences d'expression in vitro des ARNm correspondant aux sousunités alpha et bêta du récepteur GABA/benzodiazépine identifiées jusque-là ont montré que ces deux sous-unités ne suffisaient pas à elles seules pour former un complexe récepteur parfaitement semblable au récepteur natif ; en effet, le complexe récepteur $\mathrm{GABA} /$ benzodiazépine était jusqu'ici considéré comme un simple tétramère de type (alpha) ${ }_{2}$ (bêta) dans lequel la sous-unité alpha représente le site de liaison des benzodiazépines et la sous-unité bêta, celui du GABA [1, 31]. Les auteurs ont poursuivi l'examen systématique des banques d'ADNc (avec les mêmes sondes ou d'autres) et deux nouvelles sous-unités ont été mises en évidence : gamma ${ }^{2}$ et delta. La sousunité gamma ${ }^{2}$, neuronale (la sousunité gamma ${ }^{1}$ étant gliale) est indispensable à la liaison des benzodiazé- pines [32] tandis que la sous-unité delta semble plus spécifique du GABA [33].

Ces quatre sous-unités présentent des régions homologues de plus ou moins grande importance et des caractéristiques structurales semblables : segments trans-membranaires, sites de glycosylation, position du site récepteur en région $\mathrm{N}$-terminale...). Les résultats obtenus dans les diverses expérimentations (expression in vivo suivie d'études électrophysiologiques ou de caractérisation de la liaison spécifique de plusieurs ligands) montrent la meilleure fonctionnalité de ce type de complexe. Une structure pentamérique doit donc à présent être admise, de type (alpha) $)_{2}$, (bêta) ${ }_{2}$ $\left(\text { gamma }^{2}\right)_{1}$ ou $(\text { alpha })_{2}$ (bêta $)_{2}$, (delta) pour le complexe récepteur $\mathrm{GABA}_{\mathrm{A}}$ /benzodiazépine. Le nombre de ces combinaisons est encore augmenté en raison de l'existence de plusieurs sous-unités alpha et bêta.

\section{Corrélations anatomiques et histologiques des récepteurs GABA et benzodiazépine dans le cervelet : récepteurs couplés et non couplés}

Les déterminations faites sur des homogénats ou des préparations membranaires donnent des valeurs globales alors que dans le système nerveux central on a affaire à des circuits complexes; pour mieux comprendre les interactions précises entre les récepteurs GABA et benzodiazépine, il est important de les localiser dans le tissu afin de compléter les données pharmacologiques obtenues précédemment. La distribution d'un récepteur peut être visualisée par des techniques autoradiographiques, immunohistologiques ou issues de la biologie moléculaire, chacune d'elles ayant ses spécificités et ses limites. Par l'autoradiographie, c'est le ligand fixé sur son récepteur spécifique qui est visualisé. Cette méthode permet de localiser les sites récepteurs, soit en microscopie optique (qui permet aussi leur quantification), soit en microscopie électronique (qui donne la localisation sub-cellulaire [34]). C'est surtout le muscimol, agoniste du GABA, qui a été utilisé comme ligand pour les marquages autoradio- 
graphiques du site $\mathrm{GABA}_{\mathrm{A}}$, cependant que la grande majorité des études visant à localiser le site benzodiazépine ont été réalisées avec le flunitrazépam [3, 35-37]. Dans l'étude immunohistochimique, c'est le récepteur qui est visualisé dans le tissu nerveux par son association avec des anticorps (monoclonaux le plus souvent) dirigés contre le complexe récepteur GABA/benzodiazépine isolé du cortex cérébral de bœuf. La plupart des études visant à localiser le récepteur GABA/benzodiazépine ont été menées avec des anticorps monoclonaux (MAb) nommés respectivement bd17 et bd24 [31, 38]. Le MAb bd17 reconnaît la sous-unité bêta du récepteur $\mathrm{GABA}_{\mathrm{A}} /$ benzodiazépine dans toutes les espèces testées tandis que le MAb bd24 reconnaît la sous-unité alpha du récepteur $\mathrm{GABA}_{\mathrm{A}}$ /benzodiazépine (sauf chez le rat chez qui il ne réagit pas) $[31,38$, 39]. D'autres anticorps ont également été utilisés [40-42].

Il ressort des études autoradiographiques que les récepteurs $\mathrm{GABA}_{\mathrm{A}}$ et benzodiazépine ne seraient pas systématiquement présents simultanément

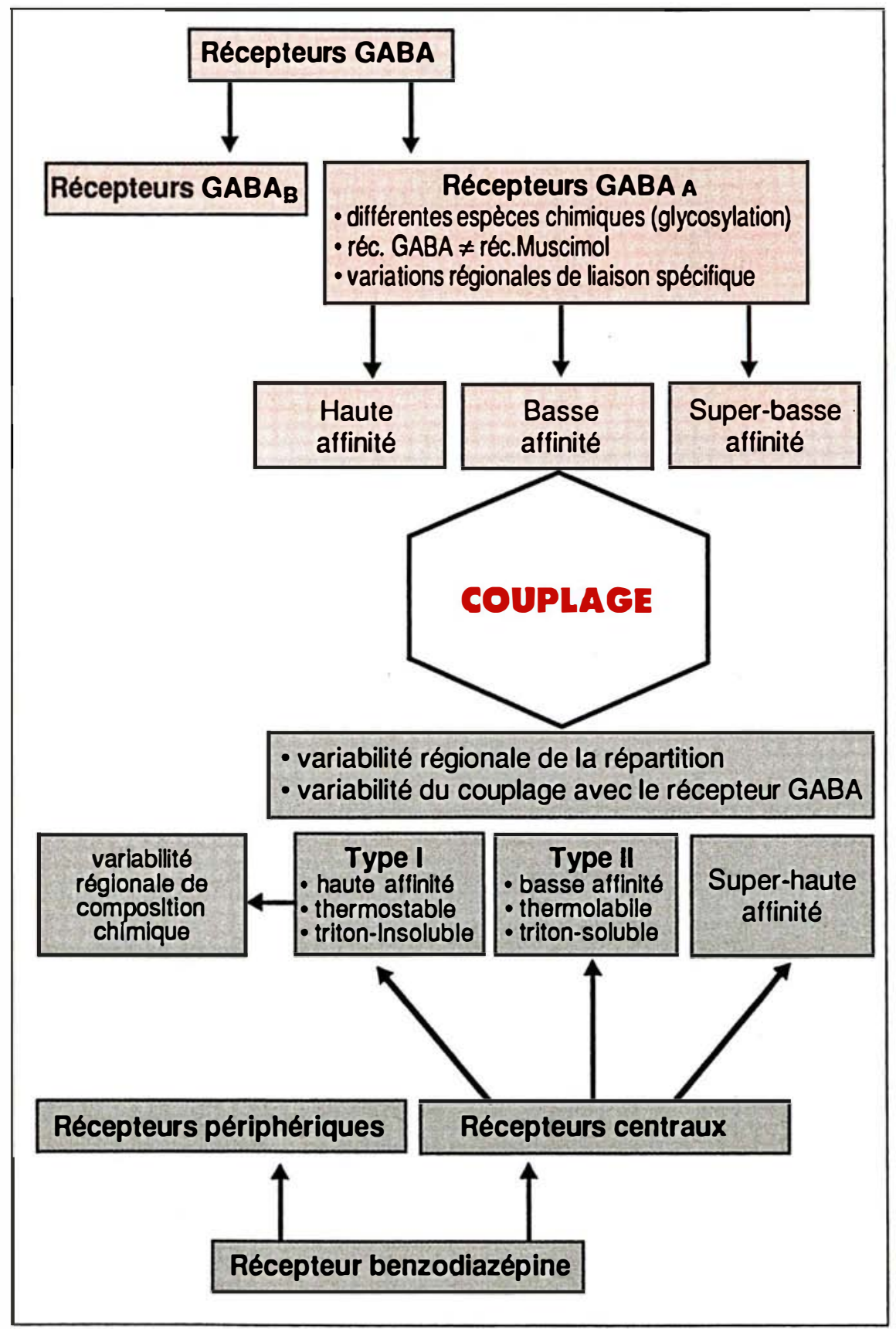

Figure 3. Hétérogénéité des récepteurs GABA et des récepteurs benzodiazépine et corrélations anatomo-histologiques : récepteurs couplés et non couplés. L'activité inhibitrice due au GABA est transmise par deux sous-types de récepteurs, les récepteurs $G A B A_{A}$ et $G A B A_{B}$, qui se distinguent par leur mode d'association à un canal ionique. Le récepteur $G_{A B A_{A}}$ est lui-même constitué de trois sous-classes $d^{\prime}$ affinités différentes. II ressort des études autoradiographiques et immunohistologiques que les récepteurs $G_{A B A_{A}}$ et benzodiazépine ne sont pas systématiquement présents simultanément dans une même zone et que ce sont les récepteurs $G_{A B A_{A}}$ à basse affinité qui sont impliqués lorsqu'il y a couplage. Les récepteurs benzodiazépine sont classés en sites "centraux" (neuronaux) et "périphériques" (essentiellement gliaux). Les récepteurs benzodiazépine centraux se divisent euxmêmes en trois sous-groupes en fonction des affinités de leurs ligands spécifiques. Ces sous-types présentent des différences régionales dans leur association avec le récepteur $G A B A_{A}$, introduisant par là un degré supplémentaire d'hétérogénéité qui s'additionne à $d^{\prime}$ autres subtiles variations biochimiques et physiologiques. Les données.structurales et anatomiques obtenues grâce aux techniques de biologie moléculaire permettent de fournir une explication quant à la multiplicité des sous-classes et à l'hétérogénéité anatomique de leurs couplages. 


\section{RÉFÉRENCES}

34. Kuhar MJ. Receptor localization with the microscope. In: Yamamura HI, Enna SJ, Kuhar MJ, eds. Neurotransmitter Receptor Binding. New York : Raven Press, 1985 ; 153-76.

35. Unnerstall JR, Kuhar MJ, Niehoff DL, Palacios JM. Benzodiazepine receptors are coupled to a subpopulation of gammaaminobutyric acid (GABA) receptors : evidence from a quantitative autoradiographic study. J Pharmacol Exp Therap 1981 ; 218 : 797-804.

36. McCabe RT, Wamsley JK. Autoradiographic localization of subcomponents of the macromolecular GABA receptor complex. Life Sci 1986 ; 39 : 1937-45.

37. Palacios JM, Wamsley JK, Kuhar MJ. High affinity GABA receptors-autoradiographic localization. Brain Res 1981 ; 222 : 285-307.

38. Richards JG, Schoch $P$, Häring $P$, Takacs B, Möhler $H$. Resolving $\mathrm{GABA}_{\mathrm{A}}$ /benzodiazepine receptors : cellular and subcellular localization in the CNS with monoclonal antibodies. J Neurosci 1987 ; 7 : 1866-86.

39. Somogyi P, Takagi H, Richards JG, Möhler H. Subcellular localization of benzodiazepine/GABA $\mathrm{G}_{\mathrm{A}}$ receptors in the cerebellum of rat, cat, and monkey using monoclonal antibodies. J Neurosci $1989 ; 9$ : 2197-209. 40. Fuchs K, Möhler $\mathrm{H}$, Sieghart W. Various proteins from rat brain, specifically and irreversibly labeled by $\left[{ }^{3} \mathrm{H}\right]$ flunitrazepam, are distinct alpha-subunits of the GABA-benzodiazepine receptor complex. Neurosci Lett 1988 ; 90 : 314-9.

41. De Blas AL, Vitorica J, Friedrich $P$ Localization of the GABA receptor in the rat brain with a monoclonal antibody to the $57,000 \mathrm{Mr}$ peptide of the $\mathrm{GABA}_{\mathrm{A}}$ receptor/benzodiazepine receptor $/ \mathrm{Cl}^{-}$channe complex. J Neurosci 1988 ; 8 : 602-14. 42. Meinecke DL, Tallman J, Rakic P. $\mathrm{GABA}_{\mathrm{A}}$ /benzodiazepine receptor-like immunoreactivity in rat and monkey cerebellum. Brain Res 1989 ; 493 : 303-19.

43. Olsen RW, Snowhill EW, Wamsley JK. Autoradiographic localization of low affinity GABA receptors with $\left[{ }^{3} \mathrm{H}\right]$ bicuculline methochloride. Eur J Pharmacol 1984 ; 99 : 247-8.

44. Olsen RW, McCabe RT, Wamsley JK. $\mathrm{Gaba}_{\mathrm{A}}$ receptor subtypes : autoradiographic comparison of GABA, benzodiazepine, and convulsant binding sites in the rat central nervous system. J Chem Neuroanat 1990 ; 3 : 59-76.

45. Schmitz E, Reichelt R, Walkowiak W, Grayson Richards J, Hebebrand J. A comparative phylogenic study of the distribution of cerebellar $\mathrm{GABA}_{\mathrm{A}}$ /benzodiazepine receptors using radioligands and monoclonal antibodies. Brain Res 1988 ; 473 : 314-20.

46. Dutar P, Nicoll RA. A physiological role for $\mathrm{GABA}_{\mathrm{B}}$ receptors in the central nervous system. Nature 1988 ; 332 : 156-8. 47. Alger BE, Nicoll RA. Pharmacological evidence for two kinds of GABA receptor on rat hippocampal pyramidal cells studied in vitro. J Physiol $1982 ; 328$ : 125-41.

48. Lister RG, Nutt DJ. Is RO 15-4513 a specific alcohol antagonist ? Trends Neurosci dans une même zone : ces études semblent en effet révéler une dissociation anatomique des récepteurs $\mathrm{GABA}_{\mathrm{A}}$ et benzodiazépine $[5,20$, $35,37]$ qui seraient couplés, le cas échéant, à la seule fraction de récepteurs $\mathrm{GABA}_{\mathrm{A}}$ à basse affinité [43, 44].

Les expériences d'immunoprécipitation avec les anticorps monoclonaux spécifiques des sous-unités alpha et bêta du récepteur montrent que chacun des deux anticorps est capable d'immuno-précipiter le complexe récepteur entier, ce qui suggère une coexistence étroite des sites de liaison du GABA et des benzodiazépines [38, 41, 45].

Les données récentes obtenues par les techniques de biologie moléculaire apportent un éclairage nouveau sur le complexe récepteur GABA/benzodiazépine. L'application sur des tranches de cerveau de rat de $\left[{ }^{35} \mathrm{~S}\right] \mathrm{ARNm}$ transcrits à partir d'ADNc codant pour les sous-unités du récepteur permet de révéler la localisation des gènes correspondants [28, 29]. L'hybridation in situ de tranches de cervelet de rat avec le $\left[{ }^{35} \mathrm{~S}\right] \mathrm{ARNc}$ correspondant à la sousunité gamma ${ }^{2}$ donne le même marquage que l'autoradiographie par les benzodiazépines [32], cependant que les expériences réalisées avec l'ADNc codant pour la sous-unité delta révèlent un marquage similaire à celui obtenu avec le muscimol [33, 35, 37]. A partir des deux modèles contenant soit la sous-unité gamma ${ }^{2}$, soit la sous-unité delta, les " thèmes et variations " dûs aux sous-types alpha ${ }^{1}$, alpha ${ }^{2}$, alpha $^{3}$, bêta ${ }^{1}$ et bêta ${ }^{2}$ pourraient être corrélés au grand nombre de types de récepteurs $\mathrm{GABA}_{\mathrm{A}}$ /benzodiazépine (haute et basse affinité, couplage du récepteur GABA aux récepteurs benzodiazépine de type I ou II...) et sont certainement à la base des contradictions apparentes observées tant en histoimmunologie ou histo-autoradiographie qu'en hybridation in situ.

\section{Effets physiologiques}

\section{- Récepteurs GABA}

Les études électrophysiologiques montrent que la stimulation des récepteurs GABA entraîne une inhi- bition de l'activité neuronale. Cet effet peut être à médiation pré- ou post-synaptique et impliqué par des récepteurs $\mathrm{GABA}_{\mathrm{A}}$ ou $\mathrm{GABA}_{\mathrm{B}}$ [1]. Quelques nuances s'imposent toutefois : si le $\mathrm{GABA}_{\mathrm{B}}$ potassique est bien hyperpolarisant, les changements de conductance qu'il induit sont faibles et sa fonction n'est probablement pas stricto sensu inhibitrice [46]. Par ailleurs, bien que le $\mathrm{GABA}_{\mathrm{A}}$ soit généralement inhibiteur, deux types de réponses peuvent être observées au cours d'expériences électrophysiologiques consécutivement à l'application de GABA sur des tranches d'hippocampe : c'est ainsi qu'appliqué au niveau du soma, il entraîne une hyperpolarisation alors que c'est une dépolarisation qui est mesurée lorsqu'il est appliqué au niveau des dendrites [47].

L'action inhibitrice du GABA peut entraîner au niveau d'un système donné soit une inhibition, soit une excitation globale : l'inhibition par le GABA de neurones inhibiteurs aboutira à une activation de ce système et inversement. Le GABA est impliqué dans des interactions complexes et à des niveaux multiples avec de nombreux autres systèmes neurotransmetteurs et hormonaux. Ainsi le GABA interagit avec l'acétylcholine, l'adénosine, l'angiotensine, les catécholamines, les opiacés... [1]. De telles interactions gènent l'interprétation du rôle du GABA dans de nombreux effets comportementaux étant donné qu'il peut intervenir de façon secondaire dans l'ajustement de l'action d'autres neurotransmetteurs sans avoir lui-même un rôle crucial pour la production de ces comportements. Le fait que le récepteur $\mathrm{GABA}_{\mathrm{A}}$ soit quasiment ubiquitaire dans le système nerveux central et que le récepteur $\mathrm{GABA}_{\mathrm{B}}$ puisse être présent dans les mêmes zones rend difficile l'attribution spécifique à l'un ou l'autre de ces récepteurs d'un effet donné du GABA. Par exemple, les récepteurs $\mathrm{GABA}_{\mathrm{A}}$ et $\mathrm{GABA}_{\mathrm{B}}$ sont tous deux capables d'influencer l'analgésie produite par l'intermédiaire d'autres systèmes neurotransmetteurs et par ailleurs, le GABA produit directement une analgésie par des mécanismes pré- et postsynaptiques à différents niveaux du cerveau et de la moelle épinière, cet 
effet semblant faire intervenir principalement les récepteurs $\mathrm{GABA}_{\mathrm{B}}$. Quoique les deux types de récepteurs puissent avoir des fonctions similaires, il est cependant évident qu'ils possèdent des profils physiologiques et comportementaux différents. Les récepteurs $\mathrm{GABA}_{\mathrm{A}}$ jouent un rôle dans des phénomènes tels que la régulation de l'appétit, les effets anxiolytiques, l'activité anticonvulsivante et la régulation cardiovasculaire [7]. Les récepteurs $G_{A B A}$ seraient impliqués quant à eux dans l'analgésie, la dépression et, de même que les récepteurs $\mathrm{GABA}_{\mathrm{A}}$, dans la régulation cardiovasculaire [1].

\section{- Récepteurs benzodiazépine}

Les benzodiazépines constituent une classe de composés possédant des propriétés pharmacologiques aussi diverses que la sédation, les effets anxiolytiques ou anticonvulsivants.

Les récepteurs benzodiazépine de type II dans l'encéphale et l'hippocampe sont plus probablement impliqués dans la médiation des effets anxiolytiques des benzodiazépines que les récepteurs de type I. Cette suggestion est particulièrement attrayante si l'on considère que l'anxiété serait une fonction complexe impliquant le système limbique, centre classique de l'émotion [21]. Il reste cependant difficile d'expliquer rationnellement pourquoi le cervelet adulte est riche en récepteurs de type I (plus de $90 \%$ ) alors que cette région est plus impliquée dans la régulation de la fonction motrice que des états émotionnels. Au contraire de ce que l'on peut observer pour les systèmes récepteurs bêtaadrénergiques et muscariniques, par exemple, il semble que dans l'état actuel des connaissances, il ne soit pas possible d'attribuer précisément les différents effets pharmacologiques des benzodiazépines aux sous-classes de leurs récepteurs [11, 21].

\section{Conclusion}

On voit que le récepteur $\mathrm{GABA} / \mathrm{benzo}-$ diazépine est un système d'une grande complexité tant structurale que pharmacologique présentant de plus une importante hétérogénéité anatomique dans le système nerveux $\mathrm{m} / \mathrm{s} n^{\circ} 8$, vol. 6 , octobre 90 central. Toutefois, cette complexité se reflétant dans la multitude de sousunités et de sous-types de récepteurs identifiés à ce jour permet en réalité une plus grande finesse d'ajustement du mode d'action et des couplages respectifs aboutissant à une plus grande richesse d'adaptation du système aux divers stimuli. Il est à ce jour difficile d'assigner un rôle physiologique donné à une sousclasse précise de ces récepteurs, étant donné que la localisation joue aussi sur la nature de l'effet. La combinaison d'études pharmacologiques avec des expériences de localisation et d'expression a toutefois contribué à une meilleure compréhension de la description des récepteurs GABA et benzodiazépine, qui, tout en se complexifiant, se rationalisent pour se résoudre en un ensemble plus cohérent et fonctionnel. L'existence de différents sous-types de récepteurs des neurotransmetteurs semble constituer un principe général d'organisation utilisé par le cerveau pour transcrire des signaux chimiques en réponses fonctionnelles différentes.

Souhaitons que cette connaissance moléculaire et fonctionnelle du récepteur permette d'élucider les fonctions respectives de chacune de ces catégories de récepteurs GABA pour aboutir à l'élaboration rationnelle de nouvelles substances thérapeutiques actives, capables d'agir sur chacun des sous-types de récepteurs, ou dans une région précise du système nerveux

\section{Remerciements}

Nous tenons à remercier le docteur G. Gombos et le docteur B. Foucaud dont le rôle a été primordial dans l'élaboration de plusieurs idées présentées dans cet article.

\section{TIRÉS A PART}

E. Bacon.

\section{Summary}

Heterogeneity of the GABAbenzodiazepine receptor complex

Gamma-aminobutyric acid (GABA) is the most important inhibitory neurotransmitter in the central nervous system. It exerts its physiological activity through two subtypes of GABA receptors in the brain : $\mathrm{GABA}_{\mathrm{A}}$ and $\mathrm{GABA}_{\mathrm{B}}$ receptors. GABA $_{\wedge}$ receptors are directly associated with a $\mathrm{Cl}^{-}$channel whereas $\mathrm{GABA}_{\mathrm{B}}$ receptors are coupled to a cationic channel via a second messenger system. There is a third class of GABA receptor sites, $\mathrm{GABA}_{\mathrm{C}}$, inferred from binding studies. Two different populations of $\mathrm{GABA}_{\mathrm{A}}$ receptors can be distinguished, with high and low affinities for GABA respectively. The $\mathrm{GABA}_{\mathrm{A}}$ receptor is a complex protein with multiple recognition sites as it contains binding sites for benzodiazepines, barbiturates and convulsivants. The central benzodiazepine binding sites are constituted of two subpopulations, type I and II, with high and low affinities for the ligand. These subtypes present regional differences in their association with the $\mathrm{GABA}_{\mathrm{A}}$ receptor, thus introducing a higher level of heterogeneity, in addition to some other subtle pharmacological and biochemical differences. The histological localizations of the specific receptor subtypes have revealed that $\mathrm{GABA}_{\mathrm{A}}$ and benzodiazepine binding sites are not systematically colocalized in the brain tissue and that, in fact, it is the low affinity fraction of $\mathrm{GABA}_{\mathrm{A}}$ sites which is associated with benzodiazepine receptors. Structural and anatomical data obtained by molecular biology techniques can provide an explanation for the occurrence of multiple receptor subclasses and for the anatomical heterogeneity of their coupling : reconstructed receptor complexes identified as being of the pentameric (alpha) ${ }_{2}$ (beta) ${ }_{2}$ $\left(\text { gamma }^{2}\right)_{1}$ or $(\text { alpha })_{2}$ (beta) (delta) $)_{1}$ structure display properties very close to the native purified receptors. The various possible combinations of the four subunits and their subtypes identified so far can account for a great number of other receptor complexes. 